\title{
OSCILLATION OF THIRD-ORDER QUASI-LINEAR ADVANCED DIFFERENTIAL EQUATIONS
}

\section{JOZEF DŽURINA AND BLANKA BACULÍKOVÁ}

Abstract. The objective of this paper is to study asymptotic properties of the third-order advanced differential equation

$$
\left[r(t)\left[x^{\prime \prime}(t)\right]^{\gamma}\right]^{\prime}+q(t) x^{\gamma}[\tau(t)]=0 .
$$

We offer new oscillation criteria that really take onto account the advanced argument. Some examples are also provided to illustrate the relevance of the main results.

Mathematics subject classification (2010): 34C10, 34K11.

Keywords and phrases: third-order, advanced differential equation, oscillation.

\section{REFERENCES}

[1] R. P. Agarwal, S. L. Shien, C. C. Yeh, Oscillation criteria for second order retarded differential equations, Math. Comput. Modelling, 26, 4 (1997), 1-11.

[2] B. BACUlíKovÁ, J. DžURINA, Oscillation of third-order neutral differential equations, Math. Comput. Modelling, 52 (2010), 215-226.

[3] B. Baculíková, J. Džurina, Oscillation of third-order nonlinear differential equations, Applied Math. Letters, 24 (2011), 466-470.

[4] B. BaculíkovÁ, Properties of third order nonlinear functional differential equations with mixed arguments, Abstr. Appl. Anal., (2011), 1-15.

[5] B. BACUlíKovÁ, J. DŽURINA, Oscillation of third-order functional differential equations, EJQTDE, 43 (2010), 1-10.

[6] J. G. Dong, Oscillation behaviour of second order nonlinear neutral differential equations with deviating argument, Com. Math Appl., 59 (2010), 3710-3717.

[7] J. DžURINA, Asymptotic properties of third order delay differential equations, Czech. Math. J., 45, 120 (1995), 443-448.

[8] L. H. Erbe, Q. Kong, B. G. Zhang, Oscillation Theory for Functional Differential Equations, Marcel Dekker, New York, 1994.

[9] L. ERBE, Existence of oscillatory solutions and asymptotic behavior for a class of third order linear differential equation, Pacific J. Math., 64 (1976), 369-385.

[10] S. R. Grace, R. P. Agarwal, R. Pavani, E. Thandapani, On the oscillation of certain third order nonlinear functional differential equations, Appl. Math. Comp., 202 (2008), 102-112.

[11] P. Hartman, A. Wintner, Linear differential and difference equations with monotone solutions, Amer. J. Math. 75 (1953), 731-743.

[12] G. S. Ladde, V. Lakshmikantham, B. G. Zhang, Oscillation Theory of Differential Equations with Deviating Arguments, Marcel Dekker, New York, 1987.

[13] T. KusANO, On even-order functional differential equations with advanced and retarded arguments, J. Differ. Equations, 45 (1982), 75-84.

[14] T. KusANo, On strong oscillation of even order differential equations with advanced arguments, Hiroshima Math. J., 11 (1981), 617-620. 